\title{
Protective Effects of Huang-Lian-Jie-Du-Tang against Polymicrobial Sepsis Induced by Cecal Ligation and Puncture in Rats
}

\author{
Yufen Wei, ${ }^{1}$ Lei Shan, ${ }^{1}$ Liming Qiao, ${ }^{2}$ Runhui Liu, ${ }^{1}$ Zhenlin Hu, ${ }^{1}$ and Weidong Zhang ${ }^{1}$ \\ ${ }^{1}$ School of Pharmacy, Second Military Medical University, 325 Guohe Road, Shanghai 200433, China \\ ${ }^{2}$ Changhai Hospital, Second Military Medical University, 174 Changhai Road, Shanghai 200433, China
}

Correspondence should be addressed to Zhenlin Hu; zhenlinhu@hotmail.com and Weidong Zhang; wdzhangy@hotmail.com

Received 23 May 2013; Revised 22 October 2013; Accepted 28 October 2013

Academic Editor: Seung-Heon Hong

Copyright (C) 2013 Yufen Wei et al. This is an open access article distributed under the Creative Commons Attribution License, which permits unrestricted use, distribution, and reproduction in any medium, provided the original work is properly cited.

\begin{abstract}
Huang-Lian-Jie-Du-Tang (HLJDT) is a traditional formula that has long been used for treatment of inflammatory diseases in Traditional Chinese Medicine. In this study, we examined its protective effect against sepsis in an experimental septic model induced by cecal ligation and puncture (CLP) in rats. The results demonstrated that prophylactic administration of HLJDT protected rats from CLP-induced lethality and ameliorated CLP-induced liver and lung injury. HLJDT treatment suppressed the production of proinflammatory cytokines, including TNF- $\alpha$, IL-1, IL-6, and IL-17A, indicating HLJDT could limit excessive inflammatory responses in septic condition. In addition, HLJDT facilitated bacterial clearance by increasing phagocytic activities of peritoneal macrophages. Furthermore, HLJDT treatment reversed CLP-induced suppression of IFN- $\gamma$ expression and blocked CLP-induced increase in IL-4 expression in spleens of rats at $24 \mathrm{~h}$ after CLP, indicating that HLJDT could reverse the shift from Th1 to Th2 response and promote Th1/Th2 balance toward Th1 predominance in septic rats. Moreover, HLJDT also inhibited the expression of IL-17A and ROR- $\gamma$ t in spleens of septic rats, indicating HLJDT is able to inhibit Thl7 activation in septic condition. In conclusion, the present study demonstrated the protective effects of HLJDT against sepsis and highlighted the potential of HLJDT as a medication for septic patients.
\end{abstract}

\section{Introduction}

Sepsis is the systemic response of the host towards invading microorganisms and their toxins $[1,2]$. Despite rapid progress in medical care over the past decades, sepsis remains a leading cause of mortality in Intensive Care Units $[1,3]$. The early hallmark sign of sepsis is a whole-body inflammatory state called systemic inflammatory response syndrome (SIRS). This early phase of excessive systemic inflammation can compromise the function of distinct organ systems, leading to multiple organ dysfunction syndrome (MODS) $[1,4]$. SIRS is predominantly mediated by cytokines. It is now well established that bacterial infection leads to the activation of the cytokine network [5]. In patients with sepsis, TNF- $\alpha$ is the first proinflammatory cytokine that is released, followed by others including IL-1, IL- 6 , and IL- $8[6,7]$. TNF- $\alpha$ and IL-1 are the most important proinflammatory cytokines, they act synergistically in activating target cells and inducing the production of more inflammatory mediators and are largely responsible for the clinical manifestations of sepsis $[6,8]$. More recently, IL-17A (the first described member of the IL17 family) was identified as a novel mediator of sepsis. As an important proinflammatory cytokine, IL-17A mediates neutrophil stimulation and Tymphocyte mobilization in sepsis $[9,10]$. In addition to activating a proinflammatory cytokine cascade, inflammatory stimuli activate the production of anti-inflammatory cytokines such as IL-10, IL-4, IL-13, and TGF- $\beta[11,12]$. These cytokines suppress the expression of IL$1 \beta$ and TNF- $\alpha$, promote a switch from Th1 to Th 2 activation, and inhibit antigen presentation by monocytes as well as $\mathrm{T}$ and B lymphocyte function. In sepsis, the initial excessive inflammatory response is progressively counterbalanced by the negative feedback of anti-inflammatory process, which may adversely affect immune functions leading to inability to 
clear the infection and predisposition to secondary nosocomial infections thereby having a deleterious effect on patient outcome $[13,14]$.

On the assumption that endotoxin, proinflammatory cytokines, and other inflammatory mediators were responsible for the manifestations of sepsis, strategies were developed for blocking these inflammatory mediators $[15,16]$. However, attempts at attenuating the inflammatory response by the neutralization of individual mediators have failed [17, 18]. Clinical trials have demonstrated that the anti-inflammatory treatments with agents such as antiendotoxin antibodies [19, 20], monoclonal antibodies against $\operatorname{TNF}[21,22]$, soluble TNF receptors [23, 24], IL-1 receptor antagonist [18, 25], PAF antagonists [26, 27], or a nonselective NOS inhibitor [28] have failed to improve the outcome of patients with severe sepsis. Nevertheless, since sepsis is characterized by the release of a great variety of inflammatory mediators into the circulation, it still holds promise that combinatory therapy containing a "cocktail" of agents which modulates multiple pathogenic pathways will prove to be beneficial in the treatment of patients with sepsis [29-31]. Furthermore, the host response to sepsis involves many subsequent and concurrent processes that involve both exaggerated inflammation and immune suppression. Future therapy should emphasize both limiting systemic inflammatory response and improving host immunity against infections.

Traditional Chinese Medicine (TCM) has advocated combinatory therapeutic strategies over thousands of years. Formulae containing a combination of different kinds of herbs are often used in TCM to increase efficacy and to lower toxicity. Huang-Lian-Jie-Du-Tang (HLJDT) is a traditional formula consisting of four herbs: Rhizoma Coptidis, Radix scutellariae, Cortex Phellodendri, and Fructus Gardeniae. It has wide applications in the clinical practice of TCM. Especially, HLJDT is recognized in China as an effective anti-inflammatory agent and has been widely used in the treatment of various inflammatory diseases such as gastritis, dermatitis, and aphthous stomatitis [32]. Animal studies demonstrated that treatment with HLJDT could provide therapeutic efficacy in various inflammatory models, such as dextran sulfate sodium-induced colitis in mice [33], collageninduced arthritis in rats $[34,35]$, and carrageenan-induced paw edema in mice [36]. In animal experiments, HLJDT suppressed the secretion of proinflammatory cytokines including TNF- $\alpha$, IL-1, and IL-6 under inflammatory conditions [33]. The secretion of these cytokines was also reported to be suppressed by HLJDT in vitro [37]. In the present study, we examined whether HLJDT is of a potential therapeutic value for sepsis and investigated its anti-inflammatory and immune modulating roles in septic condition.

\section{Materials and Methods}

2.1. Medical Herbs. Rhizoma Coptidis (rhizoma of Coptis chinensis Franch), Radix Scutellariae (radix of Scutellaria baicalensis Georgi), Cortex Phellodendri (cortex of Phellodendron chinense Schneid), and Fructus Gardeniae (fructus of Gardeniajasminoides Ellis) were purchased from Bozhou
(Anhui province, China) and were authenticated by Professor HanMing Zhang (Second Military Medical University, Shanghai, China). The voucher specimens were stored in Department of Natural Medicinal Chemistry, School of Pharmacy, Second Military Medical University.

2.2. Preparation of HLJDT. HLJDT extract was prepared as previously described [38]. In brief, Rhizoma Coptidis, Radix Scutellariae, Cortex Phellodendri, and Fructus Gardeniae were mixed in a ratio of $3: 2: 2: 3$. The mixture was decocted twice with boiling water $(1: 10, \mathrm{v} / \mathrm{v})$ for $1 \mathrm{~h}$, and the extracted solution was filtered through 5 layer gauzes. The filtrations were combined and freeze-dried. The lyophilized powder was stored at $4^{\circ} \mathrm{C}$ and dissolved in sterile distilled water just before administration. The yield of HLJDT extract was $20 \%$ (w/w) of the dried herbs. The quality of HLJDT extract is monitored by chromatographic fingerprinting as described previously [38].

2.3. Animals. Male Sprague-Dawley (SD) rats (3 months old, 250-300 g) were purchased from Slacom Experimental Animal Company (Shanghai, China). All animals were acclimatized under controlled temperature $\left(20 \pm 2^{\circ} \mathrm{C}\right)$, humidity $(60 \pm 5 \%)$ and $12 \mathrm{~h}$ light/12 $\mathrm{h}$ dark cycle for 1 week before the experiment.

2.4. CLP Model of Sepsis. All animal experiments were approved by the Administrative Committee of Experimental Animal Care and Use of Second Military Medical University and conformed to the National Institute of Health guidelines on the ethical use of animals. The CLP procedure was performed as described previously [39, 40]. Briefly, rats were anesthetized with an intramuscular injection of ketamine $(75 \mathrm{mg} / \mathrm{kg}$, Yuhan Corporation, Seoul, Republic of Korea) and xylazine (20 mg/kg, Bayer, Germany), a midline laparotomy was made using minimal dissection, and the cecum was ligated just below the ileocecal valve with 20 silk, so that intestinal continuity was maintained. The antimesenteric surface of the cecum was perforated with an 18-gauge needle at two locations $1 \mathrm{~cm}$ apart and the cecum was gently compressed until fecal matter was extruded. The bowel was then returned to the abdomen and the incision was closed. At the end of the operation, all rats were resuscitated with saline, $3 \mathrm{~mL} / 100 \mathrm{~g}$, body weight, given subcutaneously. The sham-operated groups were given a laparotomy, and the cecum was manipulated but not ligated or perforated. All animals were returned to their cages with free access to food and water.

2.5. Drug Administration. Rats were randomly divided into the following groups-sham group: rats underwent the sham operation and received vehicle (sterile distilled water); CLP group: rats were subjected to CLP and received vehicle; CLP + HLJDT group: rats received oral administration of HLJDT (120 or $270 \mathrm{mg} / \mathrm{kg}$ ) $2 \mathrm{~h}$ prior to CLP.

2.6. Histological Analysis. The livers and lungs of the septic and sham-operated rats were harvested at $24 \mathrm{~h}$ after CLP. The tissue samples were fixed in $10 \%$ formalin solution, embedded 
TABLE 1: The sequences of primers used in this study.

\begin{tabular}{lll}
\hline Gene name & Forward primer sequences & Reverse primer sequences \\
\hline GAPDH & CGCATCTTCTTGTGCAGTGCCAGCC & TTGTCACAAGAGAAGGCAGCCCTGG \\
TNF- $\alpha$ & ACTGAACTTCGGGGTGATCGGT & TGGTTTGCTACGACGTGGGCTA \\
IL-1 $\beta$ & AATGCCTCGTGCTGTCTGACCCAT & CCAAGGCCACAGGGATTTTGTCGTT \\
IL-6 & ACCACTTCACAAGTCGGAGGCTT & CTGACAGTGCATCATCGCTGTTCA \\
IL-10 & AAAAGCAAGGCAGTGGAGCAGGTG & TGGCCTTGTAGACACCTTTGTCTTG \\
IL-17A & ACCTCAACCGTTCCACTTCACCCT & ATGTGGTGGTCCAACTTCCCCTCA \\
IFN- $\gamma$ & GACAACCAGGCCATCAGCAACAACA & CAGCTTTGTGCTGGATCTGTGGGT \\
IL- 4 & GAACAAGTCTGGGGTTCTCG & TTGTGAGCGTGGACTCATTC \\
ROR- $\gamma \mathrm{t}$ & TCTGGAAGCTGTGGGATAGA & GAGGAGCCTGTGGAGAAATAC \\
\hline
\end{tabular}

in paraffin, and sectioned. The tissue sections were then stained with the hematoxylin and eosin reagent according to standard protocols and observed under light microscopy.

\subsection{Measurement of the Plasma Levels of ALT, AST, and} Cytokines. Blood samples were collected via the abdominal artery from the septic or sham-operated rats at $4 \mathrm{~h}, 12 \mathrm{~h}$, and $24 \mathrm{~h}$ after surgery. Eighteen rats in each group were used for blood sampling, and 6 rats were used at each time point. To collect the blood samples, rats were anesthetized with an intramuscular injection of ketamine $(75 \mathrm{mg} / \mathrm{kg}$, Yuhan Corporation, Seoul, Republic of Korea) and xylazine $(20 \mathrm{mg} / \mathrm{kg}$, Bayer, Germany). A midline laparotomy was made and the abdominal artery was exposed by shifting the intestines over to the left. The blood was collected via the abdominal artery using a 23-25-gauge needle and a $5 \mathrm{~mL}$ syringe by inserting the needle into the artery and drawing blood slowly. Plasma was obtained after centrifugation of the samples ( $300 \mathrm{~g}$ for $5 \mathrm{~min}$ ). ALT and AST levels were measured using commercialized kits purchased from Nanjing Jiancheng Bioengineering Institute (Nanjing, China) according to manufacturer's instructions. Levels of TNF- $\alpha$, IL-1, IL-6, IL-17A, and IL-10 were measured using ELISA kits purchased from eBioscience (San Diego, USA) according to manufacturer's instructions.

2.8. Analysis of Cytokine Gene Expression by Quantitative RT-PCR ( $q R T-P C T)$. Rats were sacrificed $24 \mathrm{~h}$ after CLP. The liver, lung, kidney, and spleen were harvested for RNA isolation. Total RNA was extracted with TRIzol reagent (Invitrogen, Carlsbad, CA, USA) according to manufacturer's instructions. For each sample, $500 \mathrm{ng}$ of total RNA was reversely transcribed using PrimeScript RT reagent Kit (Takara, Dalian, China). PCR amplification was performed on a StepOnePlusTM real time PCR system (Applied Biosystems, USA) using the SYBR Premix Ex TaqTM PCR Kit (Takara, Dalian, China). The primers used were designed using Primer 3 and custom-synthesized at Invitrogen (their sequences are shown in Table 1). The relative levels of assayed mRNAs were calculated with comparative Ct method using GAPDH expressions as endogenous control and were normalized to sham control.
2.9. Evaluation of Bacterial Clearance. The bacterial loads were assessed in peritoneal cavity, liver, and lung to evaluate the bacterial clearance using a method described previously [41]. Briefly, the rats were anesthetized with ketamine and xylazine at $24 \mathrm{~h}$ after CLP. The peritoneal cavities were washed with $1 \mathrm{~mL}$ of sterile PBS and the peritoneal lavage fluids were collected under sterile condition. The livers and lungs were also removed under sterile condition. The tissues obtained were individually weighed and homogenized with sterile saline. The peritoneal fluid, liver, and lung homogenates were serially diluted with sterile PBS. $30 \mu \mathrm{L}$ of each diluted sample was placed on blood-agar base plates (Trypticase Soy Agar Deeps, Becton Dickinson, USA) and incubated at $37^{\circ} \mathrm{C}$ for $24 \mathrm{~h}$. The numbers of bacterial colonies were then counted and expressed as colony-forming units (CFUs) per $\mathrm{mL}$ of peritoneal lavage or per mg of tissue.

2.10. Assay of Macrophage Phagocytosis. The peritoneal lavage was harvested from rats at $16 \mathrm{~h}$ after CLP, and peritoneal macrophages were obtained by culturing peritoneal exudate cells for $3 \mathrm{~h}$ and removing nonadherent cells as previously described [42]. The obtained macrophages were then plated into 24-well plates (Costar) at $1 \times 10^{5}$ cells/well and cultured for $30 \mathrm{~min}$ to allow macrophages to adhere. Then the cells were incubated with carboxylate-modified fluorescent microspheres (F8827, Molecular Probe, The Netherlands) for $1 \mathrm{~h}$ according to manufacturer's instructions. The cells were then washed six times with cold PBS to eliminate uningested beads. Flow cytometry was performed using a FACSCalibur (BD Biosciences, San Jose, CA, USA) and the data were analyzed with the CellQuest software package (Becton Dickinson). The percentage of phagocytic cells (PP) was defined as the percentage of macrophages that ingested one or more particles. The phagocytic index (PI) was defined as the average number of particles ingested per macrophage and was calculated as follows: the total number of ingested beads was divided by the number of total macrophages.

2.11. Statistical Analysis. All values are expressed as mean \pm SEM. All statistics analyses were performed using Prism 4.0 (GraphPad Software, USA). Differences among groups were assessed using one-way analysis of variance (ANOVA) test, 


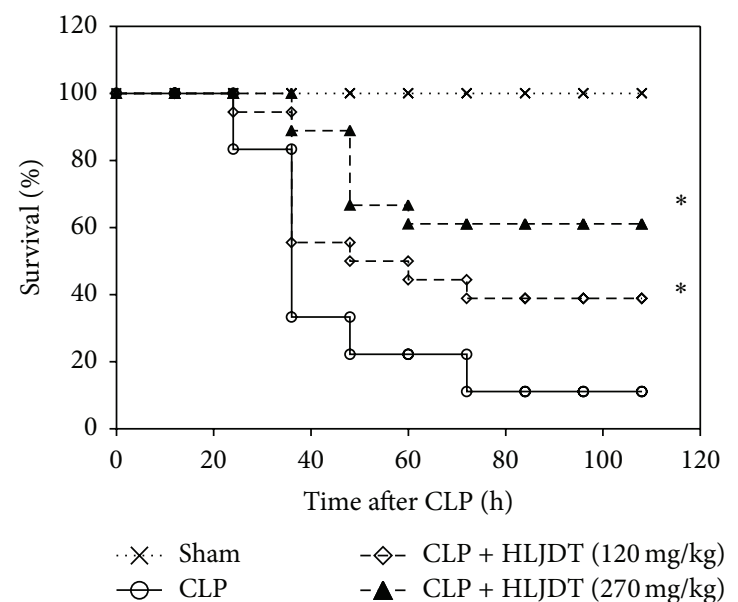

(a)

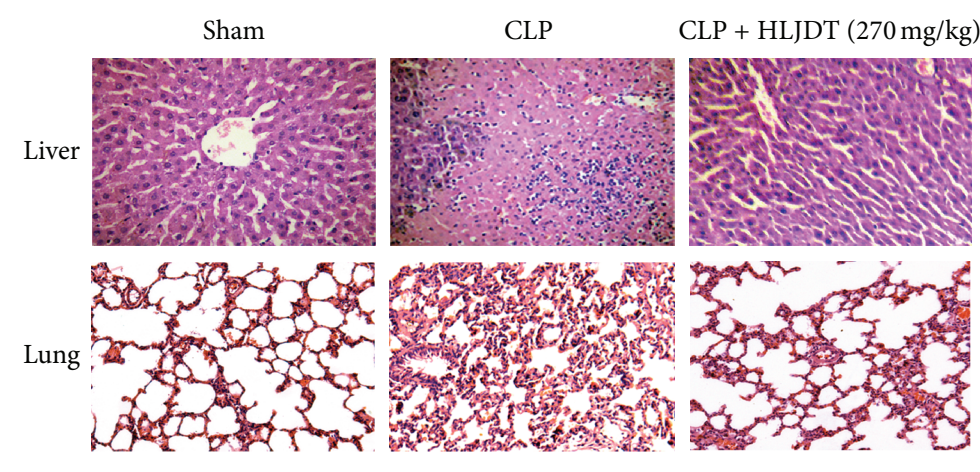

(b)

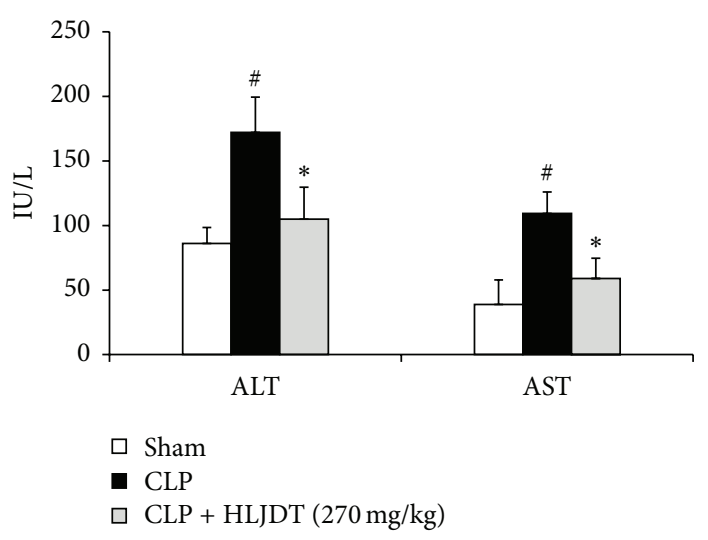

(c)

FIGURE 1: HLJDT protects rats from CLP-induced lethality and ameliorates CLP-induced tissue injuries to livers and lungs. Rats were orally administrated with HLJDT (120 or $270 \mathrm{mg} / \mathrm{kg}$ ) or vehicle at $2 \mathrm{~h}$ prior to CLP or sham surgery. (a) Survival was monitored every $12 \mathrm{~h}$ for $108 \mathrm{~h}$ after CLP. $n=18$ rats/group, ${ }^{*} P<0.05$ versus CLP control. (b) Livers and lungs were harvested 24 h after CLP for histopathologic examination using hematoxylin and eosin staining. Representative images from six animals per group were shown. (c) Blood samples were collected $24 \mathrm{~h}$ after CLP and ALT and AST levels in plasma were measured. $n=6$ rats/group, ${ }^{*} P<0.05$ versus CLP control, ${ }^{*} P<0.05$ versus sham control.

followed by Dunnett's multiple comparison post hoc test. The effect of HLJDT on survival of septic rats was analyzed by Kaplan-Meier survival curves with log-rank test. $P<0.05$ was considered statistically significant.

\section{Results and Discussion}

3.1. HLJDT Protects Rats from CLP-Induced Lethality and Ameliorates CLP-Induced Tissue Injuries to Livers and Lungs. Even though HLJDT has been shown to possess potent antiinflammatory property [32-37], its effect on sepsis has not been investigated. In this study, an experimental sepsis was induced by CLP in rats to examine the potential therapeutic value of HLJDT for sepsis. In order to evaluate protective effect of HLJDT against sepsis, rats were treated with different doses of HLJDT (120 and $270 \mathrm{mg} / \mathrm{kg}$ ) $2 \mathrm{~h}$ before CLP. Data of $108 \mathrm{~h}$ survival in HLJDT-treated and control groups are shown in Figure 1(a). Induction of sepsis by CLP resulted in
$89 \%$ mortality within $108 \mathrm{~h}$. The prophylactic administration of HLJDT resulted in a dose-dependent improvement in survival in septic rats (Figure 1(a)). Histopathological tests revealed that CLP induced marked histopathological changes such as inflammatory cell infiltration, congestion, necrosis, and degeneration in liver and lung of septic rats at $24 \mathrm{~h}$ after surgery. Administration of HLJDT $(270 \mathrm{mg} / \mathrm{kg})$ resulted in remarkable attenuation in these pathological changes (Figure 1(b)). Accordingly, CLP also caused a marked increase in the plasma levels of ALT and AST, which was significantly reduced by HLJDT treatment (Figure 1(c)). Taken together, the above results clearly demonstrated the protective effect of HLJDT against CLP-induced sepsis.

3.2. HLJDT Treatment Suppresses the Production of Proinflammatory Cytokines in Septic Rats. Exaggerated host inflammatory responses are thought to contribute to tissue injury 
and organ dysfunction in sepsis and overproduced proinflammatory cytokines, especially TNF- $\alpha$, IL-1, IL-6, and IL$17 \mathrm{~A}$, are strongly associated with sepsis syndrome. Therefore, inhibiting the overproduction of proinflammatory cytokines during early sepsis may improve sepsis outcome [3, 31, 43]. So we next investigated whether the mortality reduction by HLJDT treatment may be related to its suppression of proinflammatory cytokine production in septic rats. As shown in Figure 2(a), the plasma proinflammatory cytokines, including TNF- $\alpha$, IL-1, IL- 6 , and IL-17A, were maintained in low background levels in sham control rats within $24 \mathrm{~h}$ after surgery. In contrast, levels of TNF- $\alpha$ and IL-1 rapidly increased after CLP surgery, peaking at $4 \mathrm{~h}$, whereas the levels of IL-6 and IL-17A began to significantly increase at $12 \mathrm{~h}$ and continued to rise to a higher level till $24 \mathrm{~h}$ after CLP. The increase in TNF- $\alpha$ and IL-1 levels at 4, 12, and $24 \mathrm{~h}$ after CLP was markedly attenuated by HLJDT $(270 \mathrm{mg} / \mathrm{kg})$ treatment, and the levels of IL-6 and IL-17A at 12 and $24 \mathrm{~h}$ after CLP were also significantly suppressed by HLJDT treatment. Interestingly, even though CLP also caused a significant increase in the level of IL-10, it was not significantly affected by HLJDT treatment (Figure 2(a)). Accordingly, further determining the cytokine mRNA levels in liver, lung, and kidney by qRT-PCR revealed that CLP significantly enhanced the gene expression of these cytokines at $24 \mathrm{~h}$ after surgery, and treatment with $270 \mathrm{mg} / \mathrm{kg}$ of HLJDT resulted in a significant suppression of the expression of proinflammatory cytokines, including TNF- $\alpha$, IL-1, IL-6, and IL-17A within these organs of septic rats, whereas the enhanced expression of anti-inflammatory cytokine IL-10 was not significantly affected (Figure 2(b)). These results suggested that HLJDT can selectively suppress the expression of proinflammatory cytokines, reduce the release of these inflammatory mediators into blood circulation, and therefore attenuate the systemic inflammatory responses in septic rats.

\subsection{HLJDT Enhances Bacterial Clearance and Augments} Phagocytic Activity of Macrophage in Septic Rats. Although excessive production of systemic proinflammatory cytokines is believed to play a prominent role in the pathogenesis of tissue injury in sepsis, it should be acknowledged that these cytokines are integral components of host immunity against infections [44]. Therefore, the possibility exists that HLJDT treatment may lead to the impairment of host defense against infections and increase the risk of inability to eliminate the bacteria due to its supersession of cytokine production. To evaluate the effect of HLJDT on bacterial clearance in septic rats, we collected peritoneal lavage fluid, liver, and lung samples $24 \mathrm{~h}$ after CLP and determined the bacterial loads. The results showed that HLJDT-treated septic rats had significantly lower bacterial counts in peritoneal cavity, liver, and lung than those of untreated septic rats (Figure 3), indicating that HLJDT treatment actually enhanced bacterial clearance. Macrophages are key components of innate immunity against bacterial infections. These phagocytes mediated bacterial clearance by engulfing bacteria. We therefore further investigated whether HLJDT could affect phagocytic activity of rat macrophages using fluorescein isothiocyanate (FITC)-labeled microspheres. The results demonstrated that HLJDT significantly increased phagocytic activities of peritoneal macrophages in septic rats, as judged by percentages of fluorescent microsphere-positive cells and mean number of particles ingested per cell (phagocytic index) (Figure 4). These results suggest that the augmentation of phagocytic activities in macrophages may account, at least in part, for enhanced bacterial clearance observed in septic rats treated with HLJDT.

3.4. HLJDT Modulates Th1/Th2/Th17 Cytokine Profile in Spleens of Septic Rats. T-helper (Th) cells have long been recognized as vital components of the adaptive immune system. They convey their functions by secreting a variety of cytokines and can be subdivided into different types based on their cytokine signature. Th1 cells promote cell-mediated immune responses by secreting Thl-type cytokines such as IFN- $\gamma$ and IL-2; Th2 cells secrete cytokines such as IL-4, IL10 , and IL-5, which enhance humoral immunity $[45,46]$; Th17 cells secrete IL-17 and IL-22 which have been linked to the pathogenesis of autoimmune diseases and to immune responses to bacterial and fungal infections $[47,48]$. The effects of Th1 and Th2 cells are counterregulatory through their cytokine production. For example, IL-4 promotes Th2 development and inhibits Th1 cells. In contrast, IFN$\gamma$ promotes Thl development and inhibits the proliferation of Th2 cells and the production of IL- 4 and IL- 5 by Th2 cells. It is now well established that sepsis is associated with a profound immunological dysfunction manifested by an obvious shift from Th1 to Th2 response [49-52]. Lymphocytes from the spleens of septic mice express increased levels of Th2 cytokines, which may directly or indirectly suppress the Thl cytokine response $[53,54]$. The marked depression in cell-mediated immunity results in predisposition of septic patients to secondary infections and increased mortality in sepsis [50,51]. To investigate whether the protective effects of HLJDT in septic rats are associated with its modulation on Th1/Th2 balance in septic conditions, we examined the mRNA levels of Th1/Th2 cytokines in the spleens of HLJDTtreated and control groups. As shown in Figure 5, following CLP, the splenic IFN- $\gamma$ mRNA level increased at first at $4 \mathrm{~h}$, and declined afterwards. Till $24 \mathrm{~h}$ after CLP, it has dropped below the level in sham group (Figure 5(a)). Different from IFN- $\gamma$, the mRNA levels of IL-4 only slightly increased in spleens of septic rats within the first $12 \mathrm{~h}$ after CLP, but a dramatic increase was detected at $24 \mathrm{~h}$ after CLP. These results clearly indicated that a shift from Th1 to Th2 response was present in the septic rats following CLP (Figure 5(b)). Upon HLJDT treatment, even though the initial increase in splenic IFN- $\gamma$ mRNA level was slightly inhibited at 4 and $12 \mathrm{~h}$, the reduction of IFN- $\gamma$ level in the later phase (at $24 \mathrm{~h}$ after CLP) was reversed. At the same time, the CLPinduced increase in IL-4 expression was almost completely blocked. These data indicated that HLJDT could reverse the shift from Th1 to Th2 response and promote Th1/Th2 balance toward Thl predominance in septic rats. These data support the notion that HLJDT improved survival of septic 

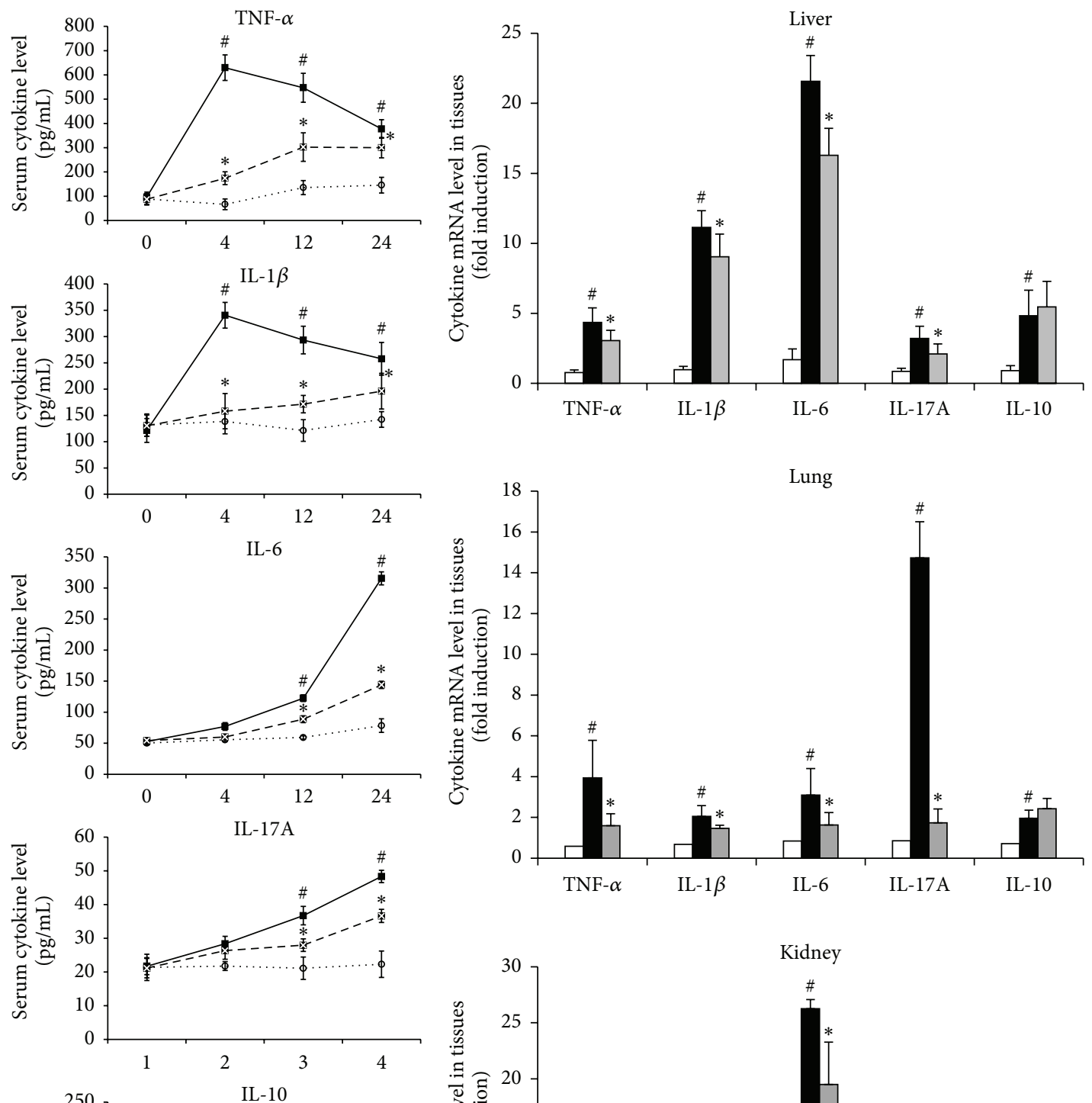

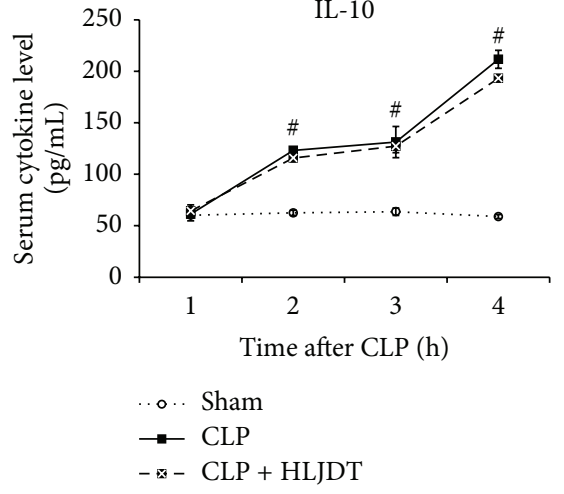

(a)

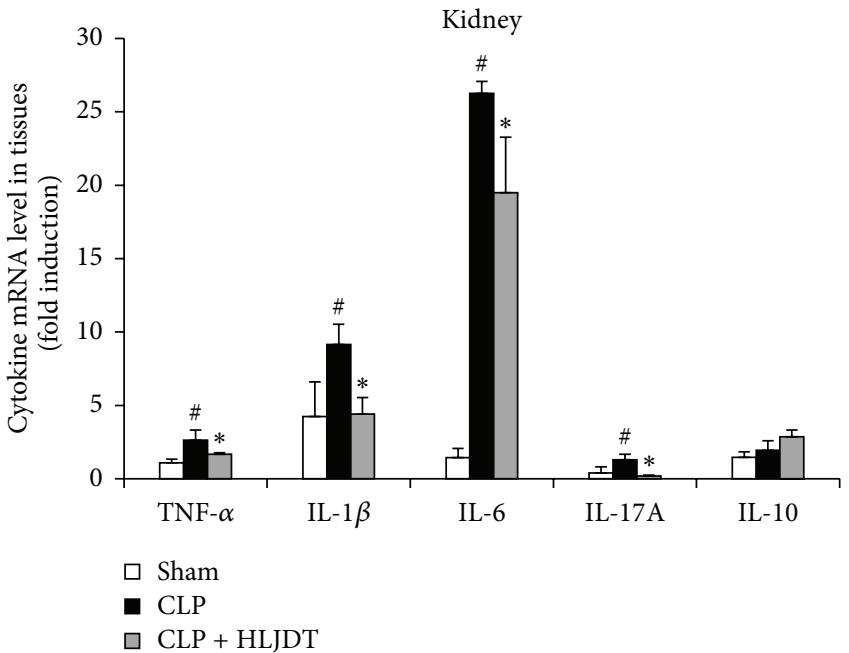

(b)

FIGURE 2: HLJDT treatment suppresses the production of proinflammatory cytokines in septic rats. Rats were orally administrated with $270 \mathrm{mg} / \mathrm{kg}$ of HLJDT or vehicle at $2 \mathrm{~h}$ prior to CLP or sham surgery. (a) Blood samples were collected at $0,4,12$, and $24 \mathrm{~h}$ after CLP, and plasma levels of TNF- $\alpha$, IL-1, IL-6, IL-17A, and IL-10 were measured with ELISA. (b) Liver, lung, and kidney were harvested at $24 \mathrm{~h}$ after CLP, and the relative mRNA levels of cytokines were analyzed by qRT-PCR. Data in (a) and (b) are mean \pm SEM of 6 rats/group, ${ }^{*} P<0.05$ versus CLP control, ${ }^{\#} P<0.05$ versus sham control. 


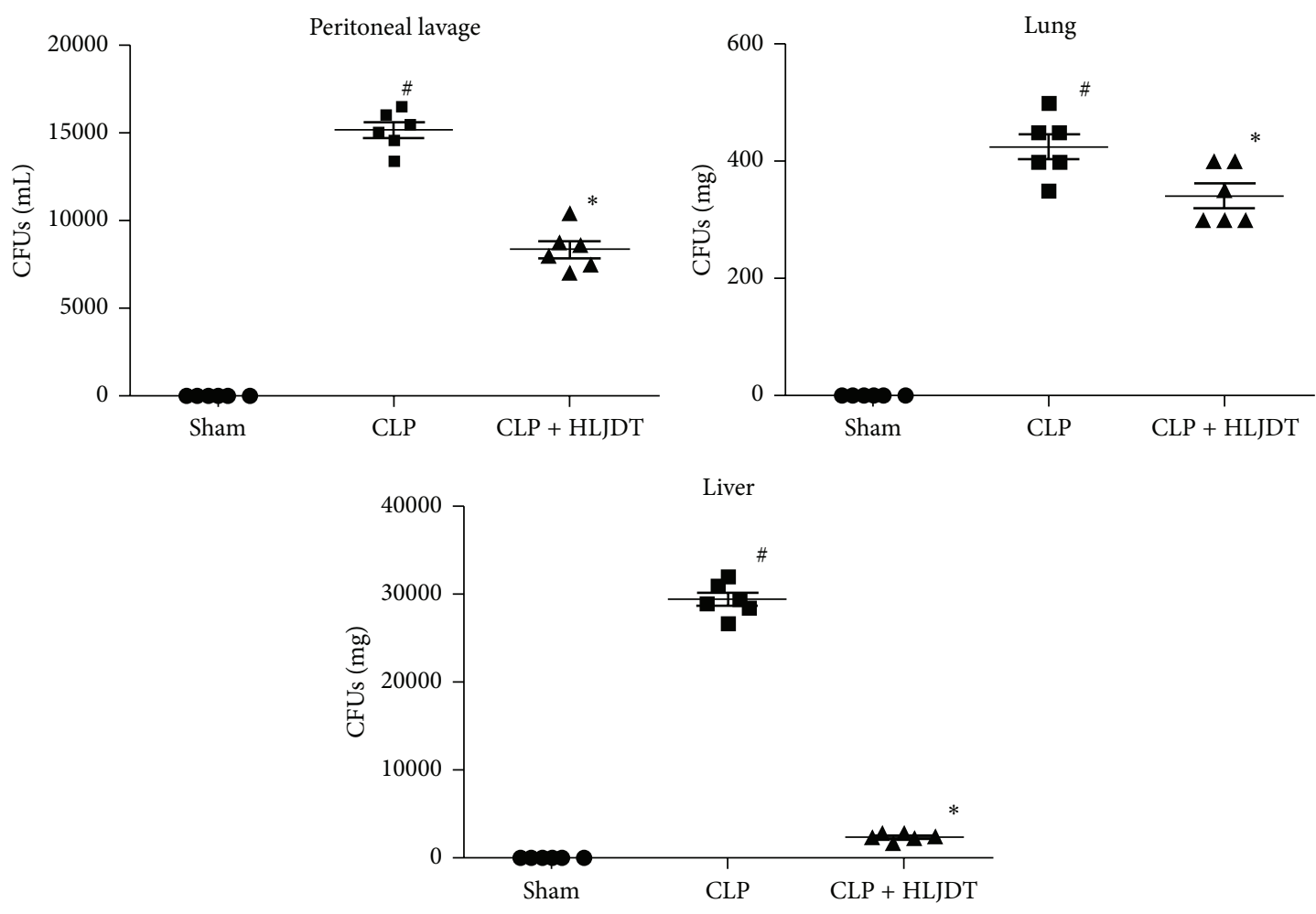

FIGURE 3: HLJDT enhances bacterial clearance in septic rats. Rats were orally administrated with $270 \mathrm{mg} / \mathrm{kg}$ of HLJDT or vehicle at $2 \mathrm{~h}$ prior to CLP or sham surgery. Peritoneal lavage fluid, liver, and lung samples were harvested $24 \mathrm{~h}$ after CLP, and the bacterial loads were determined. $n=6$ rats/group, ${ }^{*} P<0.05$ versus CLP control, ${ }^{*} P<0.05$ versus sham control.

rats as an immune-modulating agent, rather than as a simple anti-inflammatory drug. Besides Th1 and Th2 cells, Th17 cells were also found to be involved in the pathogenesis of sepsis. It was demonstrated that the CLP induced increased IL-17A production in mice and neutralization of IL-17A correlated with decreased bacteremia, increased survival, and decreased plasma levels of proinflammatory cytokines TNF$\alpha$, IL-1 $\beta$, and IL-6 [9]. In another study, CLP also increased levels of IL-22, the other hallmark cytokine of Th17 cells, in spleen and kidney of septic mice and inhibition of IL22 following CLP enhanced bacterial clearance, promoted phagocyte recruitment, and attenuated organ dysfunction [55]. In addition, clinical data from patients with abdominal sepsis suggest that higher levels of IL-22 may facilitate bacterial burden and septic complications [56]. Together, these data suggest that perturbation of Th17 activation in septic conditions negatively impacts outcomes from sepsis. In the present study, we have revealed that CLP induced a significant increase in plasma IL-17A level as well as IL-17A mRNA level in target organs including livers, lungs, and kidneys of septic rats, which were all inhibited by HLJDT treatment. These results suggest that CLP-induced Th17 activation is suppressed by HLJDT. To provide more evidence, we further detected IL-17A mRNA levels in spleens of HLJDT-treated and control groups and found that the IL-17A mRNA level progressively increased in spleens of septic rats from $4 \mathrm{~h}$ to $24 \mathrm{~h}$ following CLP, which was significantly suppressed by HLJDT treatment (Figure 5(c)). Since the transcription factor ROR- $\gamma \mathrm{t}$ is a "master gene" in controlling IL-17 and IL-22 expression in Th17 cells [48], we also examined ROR$\gamma \mathrm{t}$ mRNA level in the spleens. Accordingly, the splenic ROR- $\gamma$ t mRNA level was markedly elevated following CLP and was significantly suppressed upon HLJDT treatment (Figure 5(d)). Taken together, our data indicated HLJDT is able to inhibit the activation of Th17 cells in septic condition. Since overactivated Th17 has been proposed to have adverse functions in sepsis, it is reasonable to speculate that the protective effects of HLJDT against sepsis partially come from its inhibition of Th17.

\section{Conclusion}

In conclusion, our results showed that prophylactic administration of HLJDT protects rats from CLP-induced lethality and ameliorates CLP-induced liver and lung injury. The protective effect of HLJDT against sepsis seems to be associated with its intervention on several different aspects of sepsis, including attenuating excessive inflammatory response through suppression of the production of proinflammatory cytokines, facilitating bacterial clearance by augmentation of phagocytic activity of macrophage, preserving adaptive immune reaction by reversal of sepsis-induced shift from Th1 to Th2 response, and inhibiting Th17 activation in septic conditions. Thus, we propose that HLJDT is a potential medication for septic patients. 

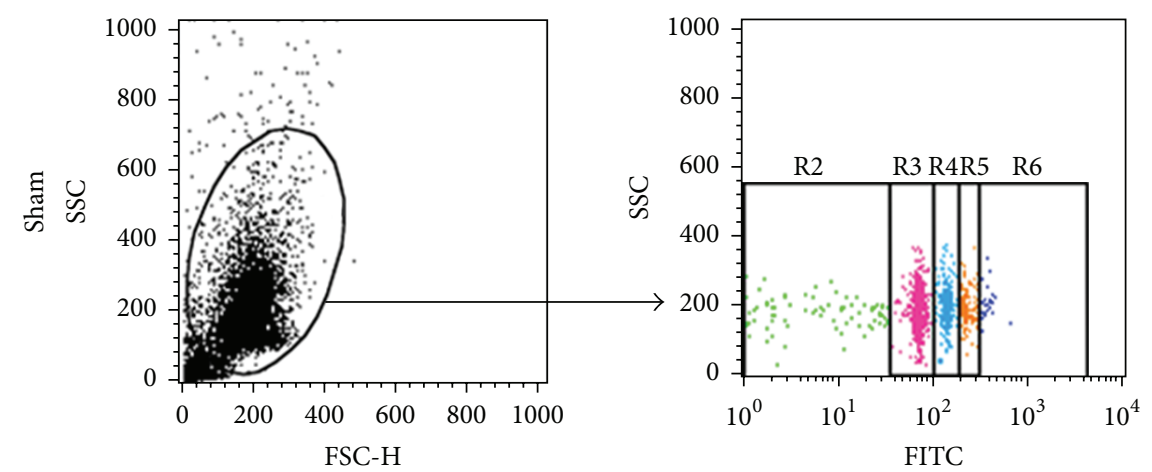

\begin{tabular}{lcc} 
Region & Events & Gate (\%) \\
\hline R2 & 5876 & 81.61 \\
R3 & 893 & 12.40 \\
R4 & 271 & 3.76 \\
R5 & 136 & 1.89 \\
R6 & 25 & 0.35 \\
R1 & 7200 & 100.00
\end{tabular}
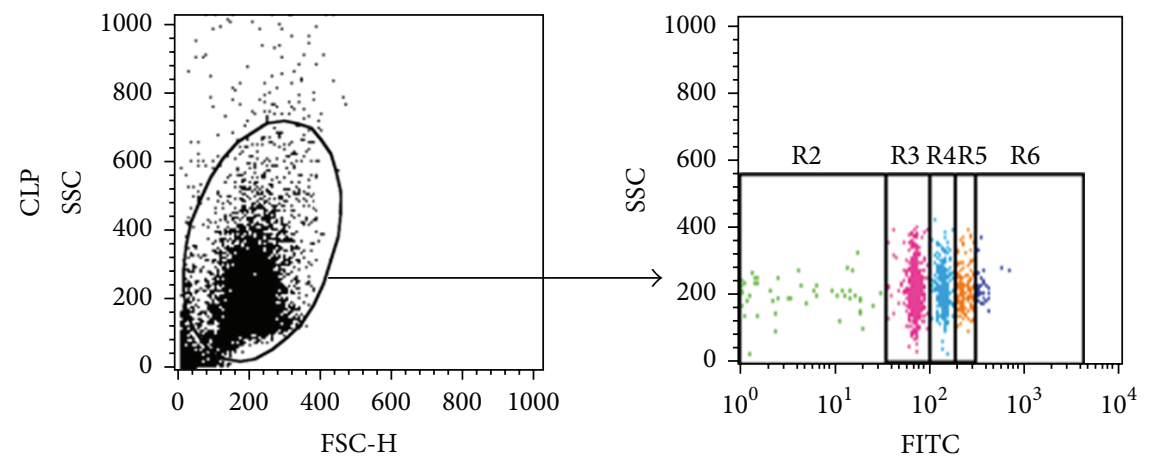

Region Events Gate $(\%)$

$\begin{array}{ccc}\text { R2 } & 5875 & 74.72 \\ \text { R3 } & 1329 & 16.90 \\ \text { R4 } & 422 & 5.37 \\ \text { R5 } & 203 & 2.58 \\ \text { R6 } & 36 & 0.46 \\ \text { R1 } & 7863 & 100.00\end{array}$
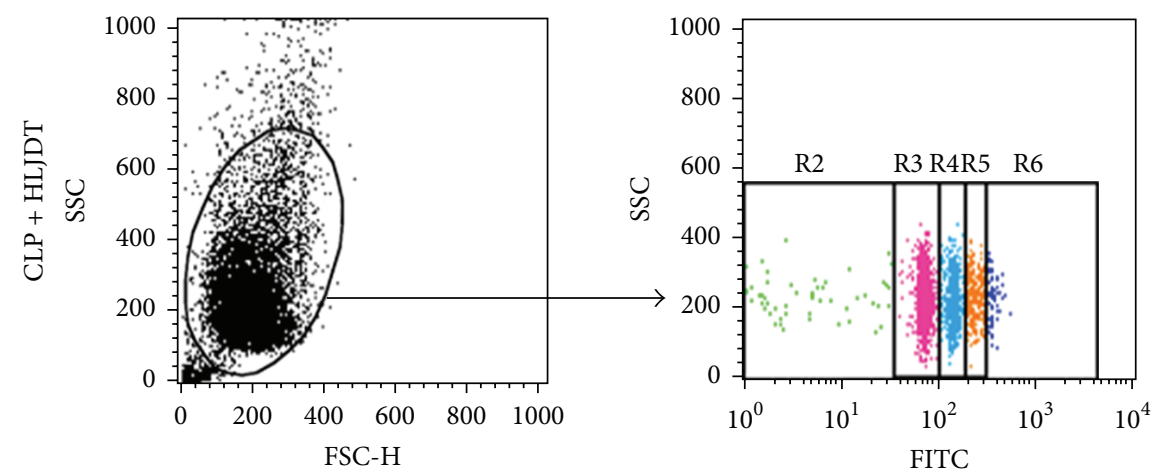

Region Events Gate (\%)

R2 $\quad 5544 \quad 67.93$

R3 $\quad 1637 \quad 20.06$

R4 $616 \quad 7.55$

R5 $\quad 310 \quad 3.80$

R6 $\quad 59 \quad 0.72$

R1 $\quad 8161 \quad 100.00$

(a)

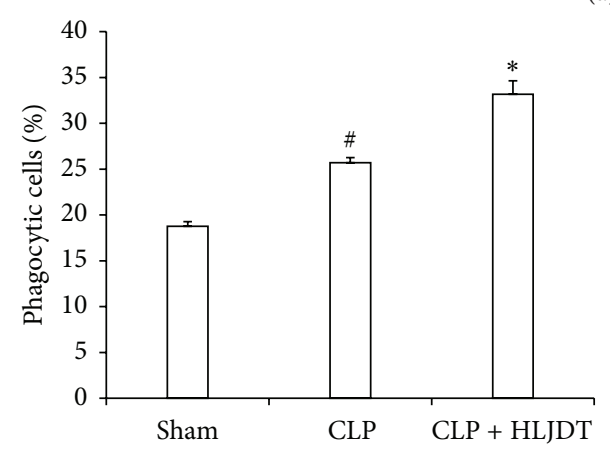

(b)

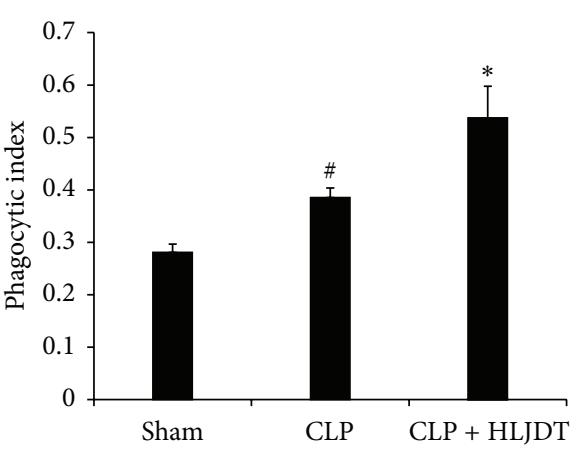

(c)

FIGURE 4: HLJDT augments phagocytic activity of macrophage in septic rats. Rats were orally administrated with $270 \mathrm{mg} / \mathrm{kg}$ of HLJDT or vehicle at $2 \mathrm{~h}$ prior to CLP or sham surgery. Phagocytotic activities of peritoneal macrophages were evaluated by uptake of FITC-conjugated microsphere at $16 \mathrm{~h}$ after CLP or sham surgery. (a) Representative results of flow cytometry analysis of phagocytosis by peritoneal macrophages after incubation with FITC-conjugated microsphere. Region 1(R1) on the dot-plot of side light scatter (SSC) versus forward light scatter (FSC) represented the gate of the macrophages. On the dot-plot of SSC versus green fluorescence (FITC), R2 represented the macrophages uningesting microspheres, and R3, R4, R5, and R6 represented the macrophages that have ingested one, two, three, and four microspheres, respectively. (b) The percentage of phagocytic cells was calculated as the percentage of macrophages that ingested one or more particles. (c) The phagocytic index was calculated as the average number of particles ingested per macrophage (the total number of ingested beads was divided by the number of total macrophages). Data in (b) and (c) are mean \pm SEM of 6 rats/group, ${ }^{*} P<0.05$ versus $\mathrm{CLP}$ control, ${ }^{*} P<0.05$ versus sham control. 


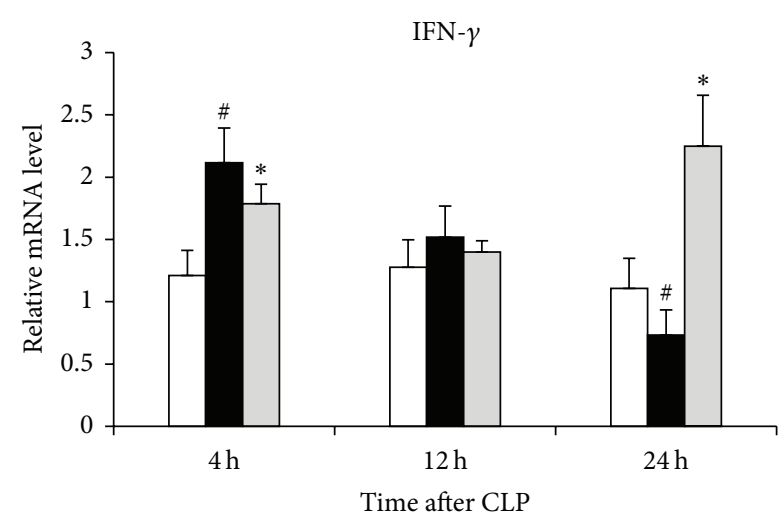

(a)

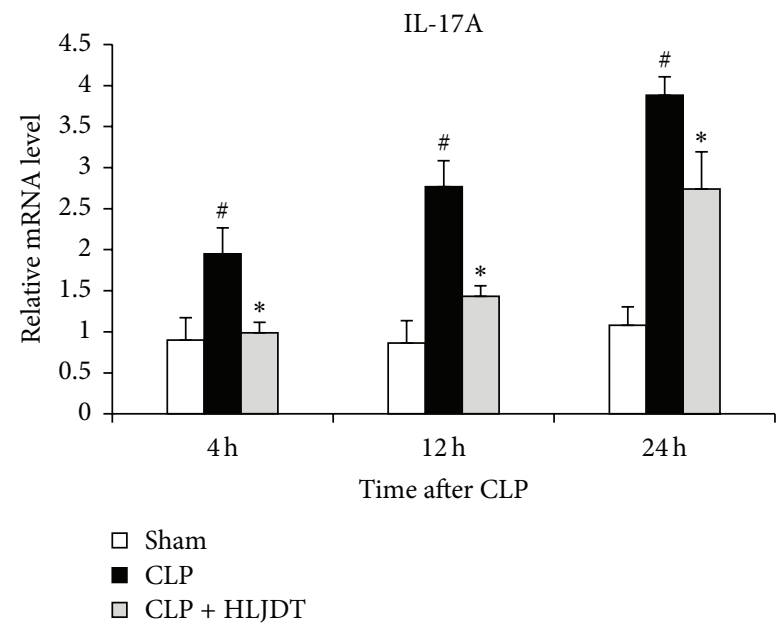

(c)

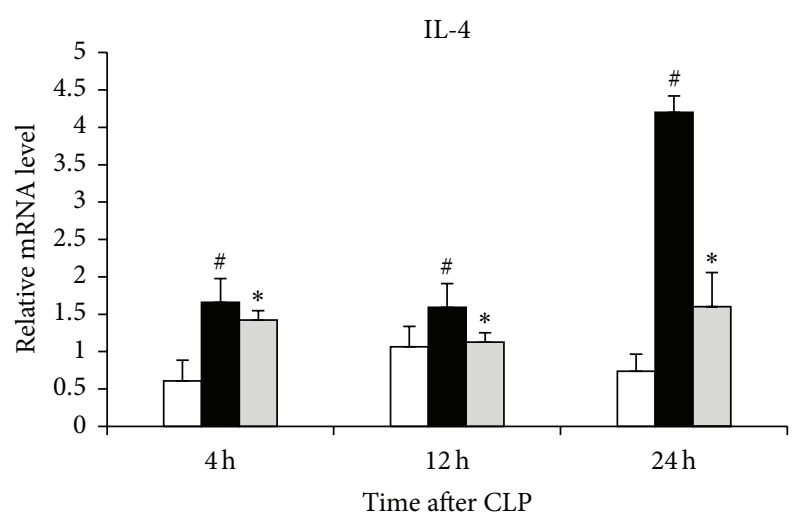

(b)

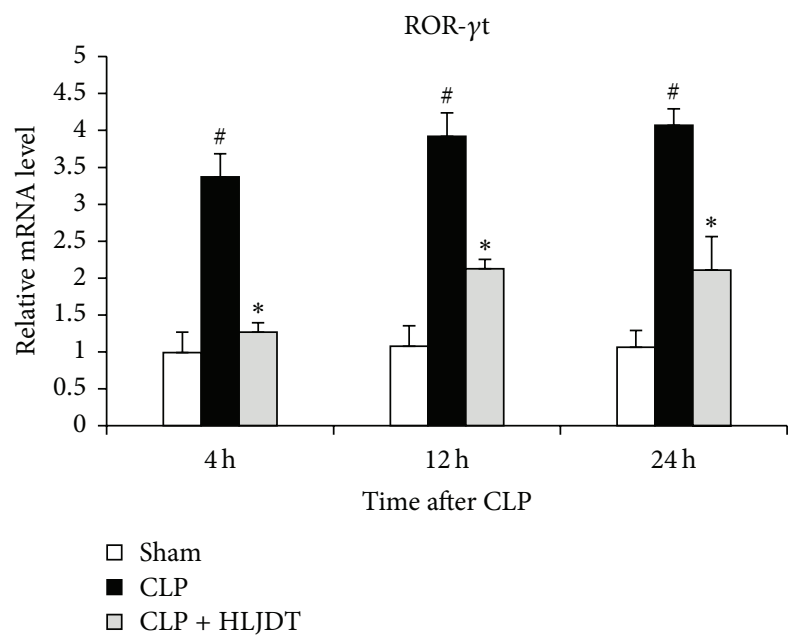

(d)

FIGURE 5: HLJDT modulates Th1/Th2/Th17 cytokine profile in spleens of septic rats. Rats were orally administrated with $270 \mathrm{mg} / \mathrm{kg}$ of HLJDT or vehicle at $2 \mathrm{~h}$ prior to CLP or sham surgery. The spleen samples were harvested at 4,12 , and $24 \mathrm{~h}$ after CLP. The relative mRNA levels of IFN- $\gamma$ (a), IL-4 (b), IL-17A (c), and ROR- $\gamma$ t (d) were analyzed by qRT-PCR. Data are mean \pm SEM of 6 rats/group, ${ }^{*} P<0.05$ versus CLP control, ${ }^{\#} P<0.05$ versus sham control.

\section{Conflict of Interests}

The authors have no financial interests, which could create a potential conflict of interests with regard to the work.

\section{Acknowledgments}

The work was supported by program NCET Foundation, NSFC (81230090), and partially supported by Global Research Network for Medicinal Plants (GRNMP) and King Saud University, Shanghai Leading Academic Discipline Project (B906), Key Laboratory of Drug Research for Special Environments, PLA, Shanghai Engineering Research Center for the Preparation of Bioactive Natural Products (10DZ2251300), the Scientific Foundation of Shanghai China (12401900801, 09DZ1975700, 09DZ1971500, and 10DZ1971700), National Major Project of China (2011ZX09307-002-03), and National Key Technology R\&D Program of China (2012BAI29B06).

\section{References}

[1] A. Lever and I. Mackenzie, "Sepsis: definition, epidemiology, and diagnosis," British Medical Journal, vol. 335, no. 7625, pp. 879-883, 2007.

[2] M. M. Levy, M. P. Fink, J. C. Marshall et al., "2001 SCCM/ ESICM/ACCP/ATS/SIS international sepsis definitions conference," Intensive Care Medicine, vol. 29, no. 4, pp. 530-538, 2003.

[3] A. P. Wheeler and G. R. Bernard, "Treating patients with severe sepsis," The New England Journal of Medicine, vol. 340, no. 3, pp. 207-214, 1999.

[4] H. K. de Jong, T. van der Poll, and W. J. Wiersinga, "The systemic pro-inflammatory response in sepsis," Journal of Innate Immunity, vol. 2, no. 5, pp. 422-430, 2010.

[5] J. T. DiPiro, "Cytokine networks with infection: mycobacterial infections, leishmaniasis, human immunodeficiency virus infection, and sepsis," Pharmacotherapy, vol. 17, no. 2, pp. 205223, 1997.

[6] C. E. Hack, L. A. Aarden, and L. G. Thijs, "Role of cytokines in sepsis," Advances in Immunology, vol. 66, pp. 101-195, 1997. 
[7] L. G. Thijs and C. E. Hack, "Time course of cytokine levels in sepsis," Intensive Care Medicine, vol. 21, no. 2, pp. S258-S263, 1995.

[8] K. J. Tracey, Y. Fong, D. G. Hesse et al., "Anti-cachectin/TNF monoclonal antibodies prevent septic shock during lethal bacteraemia," Nature, vol. 330, no. 6149, pp. 662-664, 1987.

[9] M. A. Flierl, D. Rittirsch, H. Gao et al., "Adverse functions of IL-17A in experimental sepsis," The FASEB Journal, vol. 22, no. 7, pp. 2198-2205, 2008.

[10] J. K. Kolls and A. Lindén, "Interleukin-17 family members and inflammation,” Immunity, vol. 21, no. 4, pp. 467-476, 2004.

[11] T. van der Poll, R. de Waal Malefyt, S. M. Coyle, and S. F. Lowry, "Antiinflammatory cytokine responses during clinical sepsis and experimental endotoxemia: sequential measurements of plasma soluble interleukin (IL)-1 receptor type II, IL-10, and IL13," Journal of Infectious Diseases, vol. 175, no. 1, pp. 118-122, 1997.

[12] T. Kasai, K. Inada, T. Takakuwa et al., "Anti-inflammatory cytokine levels in patients with septic shock," Research Communications in Molecular Pathology and Pharmacology, vol. 98, no. 1, pp. 34-42, 1997.

[13] C. Adrie and M. R. Pinsky, "The inflammatory balance in human sepsis," Intensive Care Medicine, vol. 26, no. 4, pp. 364$375,2000$.

[14] A. Oberholzer, C. Oberholzer, and L. L. Moldawer, "Interleukin10: a complex role in the pathogenesis of sepsis syndromes and its potential as an anti-inflammatory drug," Critical Care Medicine, vol. 30, no. 1, pp. S58-S63, 2002.

[15] K. J. Deans, M. Haley, C. Natanson, P. Q. Eichacker, and P. C. Minneci, "Novel therapies for sepsis: a review," Journal of Trauma, vol. 58, no. 4, pp. 867-874, 2005.

[16] R. S. Hotchkiss and I. E. Karl, "The pathophysiology and treatment of sepsis," The New England Journal of Medicine, vol. 348, no. 2, pp. 138-150, 2003.

[17] K. Reinhart and W. Karzai, "Anti-tumor necrosis factor therapy in sepsis: update on clinical trials and lessons learned," Critical Care Medicine, vol. 29, no. 7, pp. S121-S125, 2001.

[18] C. J. Fisher Jr., J.-F. A. Dhainaut, S. M. Opal et al., "Recombinant human interleukin 1 receptor antagonist in the treatment of patients with sepsis syndrome: results from a randomized, double-blind, placebo-controlled trial," The Journal of the American Medical Association, vol. 271, no. 23, pp. 1836-1843, 1994.

[19] R. V. McCloskey, R. C. Straube, C. Sanders, S. M. Smith, and C. R. Smith, "Treatment of septic shock with human monoclonal antibody HA-1A: a randomized, double-blind, placebocontrolled trial," Annals of Internal Medicine, vol. 121, no. 1, pp. $1-5,1994$.

[20] D. C. Angus, M. C. Birmingham, R. A. Balk et al., "E5 murine monoclonal antiendotoxin antibody in gram-negative sepsis: a randomized controlled trial," The Journal of the American Medical Association, vol. 283, no. 13, pp. 1723-1730, 2000.

[21] E. Abraham, A. Anzueto, G. Gutierrez et al., "Double-blind randomised controlled trial of monoclonal antibody to human tumour necrosis factor in treatment of septic shock," The Lancet, vol. 351, no. 9107, pp. 929-933, 1998.

[22] K. Reinhart, T. Menges, B. Gardlund et al., "Randomized, placebo-controlled trial of the anti-tumor necrosis factor antibody fragment afelimomab in hyperinflammatory response during severe sepsis: the RAMSES Study," Critical Care Medicine, vol. 29, no. 4, pp. 765-769, 2001.

[23] C. J. Fisher Jr., J. M. Agosti, S. M. Opal et al., "Treatment of septic shock with the tumor necrosis factor receptor: Fc fusion protein," The New England Journal of Medicine, vol. 334, no. 26, pp. 1697-1702, 1996.

[24] E. Abraham, M. P. Glauser, T. Butler et al., "p55 Tumor necrosis factor receptor fusion protein in the treatment of patients with severe sepsis and septic shock: a randomized controlled multicenter trial," The Journal of the American Medical Association, vol. 277, no. 19, pp. 1531-1538, 1997.

[25] S. M. Opal, C. J. Fisher Jr., J.-F. A. Dhainaut et al., "Confirmatory interleukin-1 receptor antagonist trial in severe sepsis: a phase III, randomized, double-blind, placebo-controlled, multicenter trial," Critical Care Medicine, vol. 25, no. 7, pp. 1115-1124, 1997.

[26] J.-L. Vincent, H. Spapen, J. Bakker, N. R. Webster, and L. Curtis, "Phase II multicenter clinical study of the platelet-activating factor receptor antagonist BB-882 in the treatment of sepsis," Critical Care Medicine, vol. 28, no. 3, pp. 638-642, 2000.

[27] J.-F. A. Dhainaut, A. Tenaillon, M. Hemmer et al., "Confirmatory platelet-activating factor receptor antagonist trial in patients with severe Gram-negative bacterial sepsis: a phase III, randomized, double-blind, placebo-controlled, multicenter trial," Critical Care Medicine, vol. 26, no. 12, pp. 1963-1971, 1998.

[28] A. López, J. A. Lorente, J. Steingrub et al., "Multiple-center, randomized, placebo-controlled, double-blind study of the nitric oxide synthase inhibitor 546C88: effect on survival in patients with septic shock," Critical Care Medicine, vol. 32, no. 1, pp. 21-30, 2004.

[29] A. S. Cross and S. M. Opal, "A new paradigm for the treatment of sepsis: is it time to consider combination therapy?" Annals of Internal Medicine, vol. 138, no. 6, pp. 502-505, 2003.

[30] R. P. Dellinger, J. M. Carlet, H. Masur et al., "Surviving Sepsis Campaign guidelines for management of severe sepsis and septic shock," Intensive Care Medicine, vol. 30, no. 4, pp. 536555, 2004.

[31] R. P. Wenzel, "Treating sepsis," The New England Journal of Medicine, vol. 347, no. 13, pp. 966-967, 2002.

[32] Y. Cao, C. Gao, J. Sun, J. Wang, Q. Zhou, and G. Liu, "Study on pharmacological effects of Huanglianjiedu Tang extract," Journal of China Pharmaceutical University, vol. 27, pp. 605-608, 1996.

[33] H. Tie, J. GuangBi, T. Kobayashi, S. QingHua, and C. JongChol, "Effect of Oren-gedoku-to (Huang-Lian-Jie-Du-Tang) on the murine colitis induced by dextran sulfate sodium," Journal of Traditional Medicines, vol. 17, no. 2, pp. 66-72, 2000.

[34] R. Liu, "Metabolomic study of collagen-induced arthritis in rats and the interventional effects of Huang-Lian-Jie-Du-Tang, a traditional Chinese medicine," Evidence-Based Complementary and Alternative Medicine, vol. 2013, Article ID 439690, 12 pages, 2013.

[35] R. Yue, L. Zhao, Y. Hu et al., "Rapid-resolution liquid chromatography TOF-MS for urine metabolomic analysis of collageninduced arthritis in rats and its applications," Journal of Ethnopharmacology, vol. 145, no. 2, pp. 465-475.

[36] Y. Dai, K. Miki, T. Fukuoka et al., 'Suppression of neuropeptides' mRNA expression by herbal medicines in a rat model of peripheral inflammation," Life Sciences, vol. 66, no. 1, pp. 19-29, 1999.

[37] J. Lu, J.-S. Wang, and L.-Y. Kong, "Anti-inflammatory effects of Huang-Lian-Jie-Du decoction, its two fractions and four typical compounds," Journal of Ethnopharmacology, vol. 134, no. 3, pp. 911-918, 2011.

[38] S. Dou, L. Liu, P. Jiang, W. Zhang, and R. Liu, "LC-DAD and LC-ESI-MS chromatographic fingerprinting and quantitative 
analysis for evaluation of the quality of Huang-Lian-Jie-DuTang," Chromatographia, vol. 69, no. 7-8, pp. 659-664, 2009.

[39] G. Şener, H. Toklu, F. Ercan, and G. Erkanli, "Protective effect of $\beta$-glucan against oxidative organ injury in a rat model of sepsis," International Immunopharmacology, vol. 5, no. 9, pp. 1387-1396, 2005.

[40] C. Ritter, M. Andrades, M. L. C. Frota et al., "Oxidative parameters and mortality in sepsis induced by cecal ligation and perforation," Intensive Care Medicine, vol. 29, no. 10, pp. 17821789, 2003.

[41] M. Saleh, J. C. Mathison, M. K. Wolinski et al., "Enhanced bacterial clearance and sepsis resistance in caspase-12-deficient mice," Nature, vol. 440, no. 7087, pp. 1064-1068, 2006.

[42] J. A. Steinkamp, J. S. Wilson, G. C. Saunders, and C. C. Stewart, "Phagocytosis: flow cytometric quantitation with fluorescent microspheres," Science, vol. 215, no. 4528, pp. 64-66, 1982.

[43] S. M. Opal, "The host response to endotoxin, antilipopolysaccharide strategies, and the management of severe sepsis," International Journal of Medical Microbiology, vol. 297, no. 5, pp. 365-377, 2007.

[44] M. G. Netea, J. W. M. van der Meer, M. van Deuren, and B. J. Kullberg, "Proinflammatory cytokines and sepsis syndrome: not enough, or too much of a good thing?" Trends in Immunology, vol. 24, no. 5, pp. 254-258, 2003.

[45] S. Romagnani, "Biology of human $\mathrm{T}(\mathrm{H}) 1$ and $\mathrm{T}(\mathrm{H}) 2$ cells," Journal of Clinical Immunology, vol. 15, no. 3, pp. 121-129, 1995.

[46] K. J. Tracey, B. Beutler, and S. F. Lowry, "Shock and tissue injury induced by recombinant human cachectin," Science, vol. 234, no. 4775, pp. 470-474, 1986.

[47] E. Bettelli, T. Korn, M. Oukka, and V. K. Kuchroo, "Induction and effector functions of TH17 cells," Nature, vol. 453, no. 7198, pp. 1051-1057, 2008.

[48] E. Bettelli, T. Korn, and V. K. Kuchroo, "Th17: the third member of the effector T cell trilogy," Current Opinion in Immunology, vol. 19, no. 6, pp. 652-657, 2007.

[49] N. R. Ferguson, H. F. Galley, and N. R. Webster, "T helper cell subset ratios in patients with severe sepsis," Intensive Care Medicine, vol. 25, no. 1, pp. 106-109, 1999.

[50] S. T. O’Sullivan, J. A. Lederer, A. F. Horgan et al., "Major injury leads to predominance of the T helper-2 lymphocyte phenotype and diminished interleukin-12 production associated with decreased resistance to infection," Annals of Surgery, vol. 222, no. 4, pp. 482-492, 1995.

[51] A. Ayala, Z. K. Deol, D. L. Lehman, C. D. Herdon, and I. H. Chaudry, "Polymicrobial sepsis but not low-dose endotoxin infusion causes decreased splenocyte IL-2/IFN- $\gamma$ release while increasing IL-4/IL-10 production," Journal of Surgical Research, vol. 56, no. 6, pp. 579-585, 1994.

[52] H. Iwasaka and T. Noguchi, "Th1/Th2 balance in systemic inflammatory response syndrome (SIRS)," Japanese Journal of Clinical Medicine, vol. 62, no. 12, pp. 2237-2243, 2004.

[53] W. F. Carson IV, T. Ito, M. Schaller, K. A. Cavassani, S. W. Chensue, and S. L. Kunkel, "Dysregulated cytokine expression by CD4+ T cells from post-septic mice modulates both Th1 and Th2-mediated granulomatous lung inflammation," PLOS ONE, vol. 6, no. 5, Article ID e20385, 2011.

[54] G. Y. Song, C.-S. Chung, I. H. Chaudry, and A. Ayala, "What is the role of interleukin 10 in polymicrobial sepsis: antiinflammatory agent or immunosuppressant?" Surgery, vol. 126, no. 2, pp. 378-383, 1999.
[55] G. F. Weber, S. Schlautkötter, S. Kaiser-Moore, F. Altmayr, B. Holzmann, and H. Weighardt, "Inhibition of interleukin22 attenuates bacterial load and organ failure during acute polymicrobial sepsis," Infection and Immunity, vol. 75, no. 4, pp. 1690-1697, 2007.

[56] T. M. Bingold, E. Ziesché, B. Scheller et al., "Interleukin-22 detected in patients with abdominal sepsis," Shock, vol. 34, no. 4, pp. 337-340, 2010. 


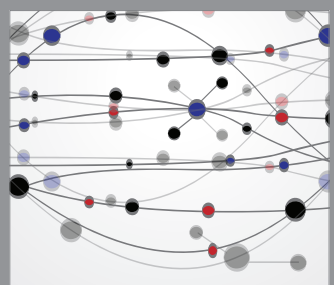

The Scientific World Journal
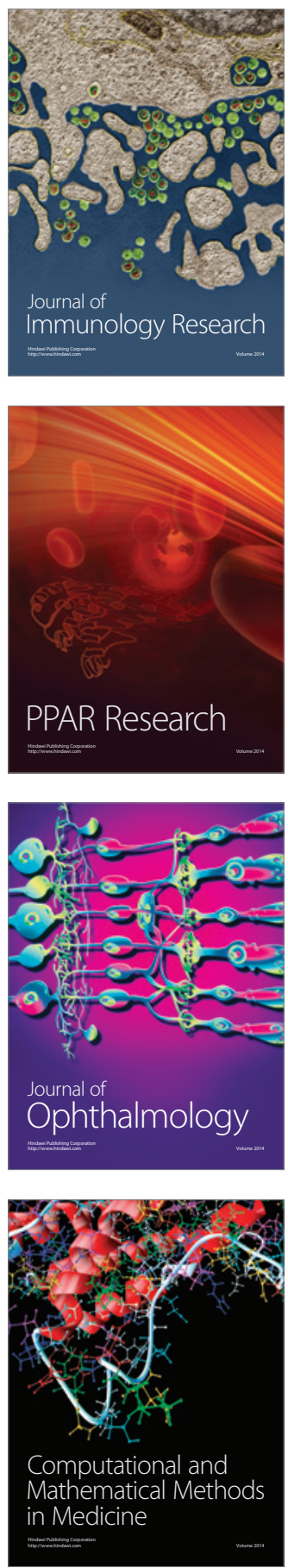

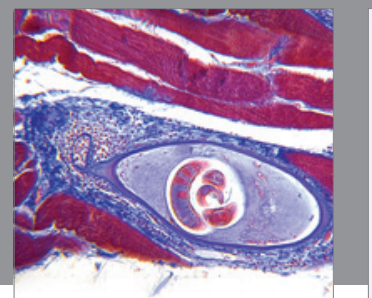

Gastroenterology

Research and Practice
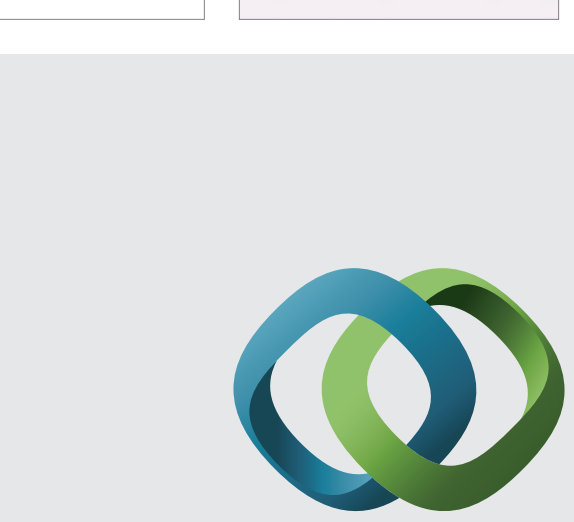

\section{Hindawi}

Submit your manuscripts at

http://www.hindawi.com
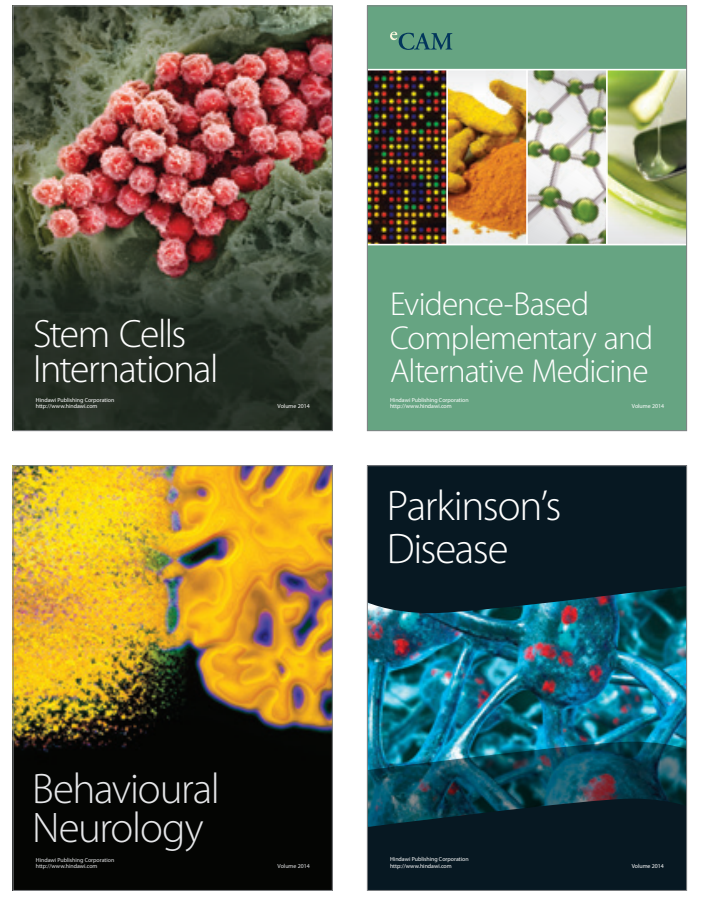
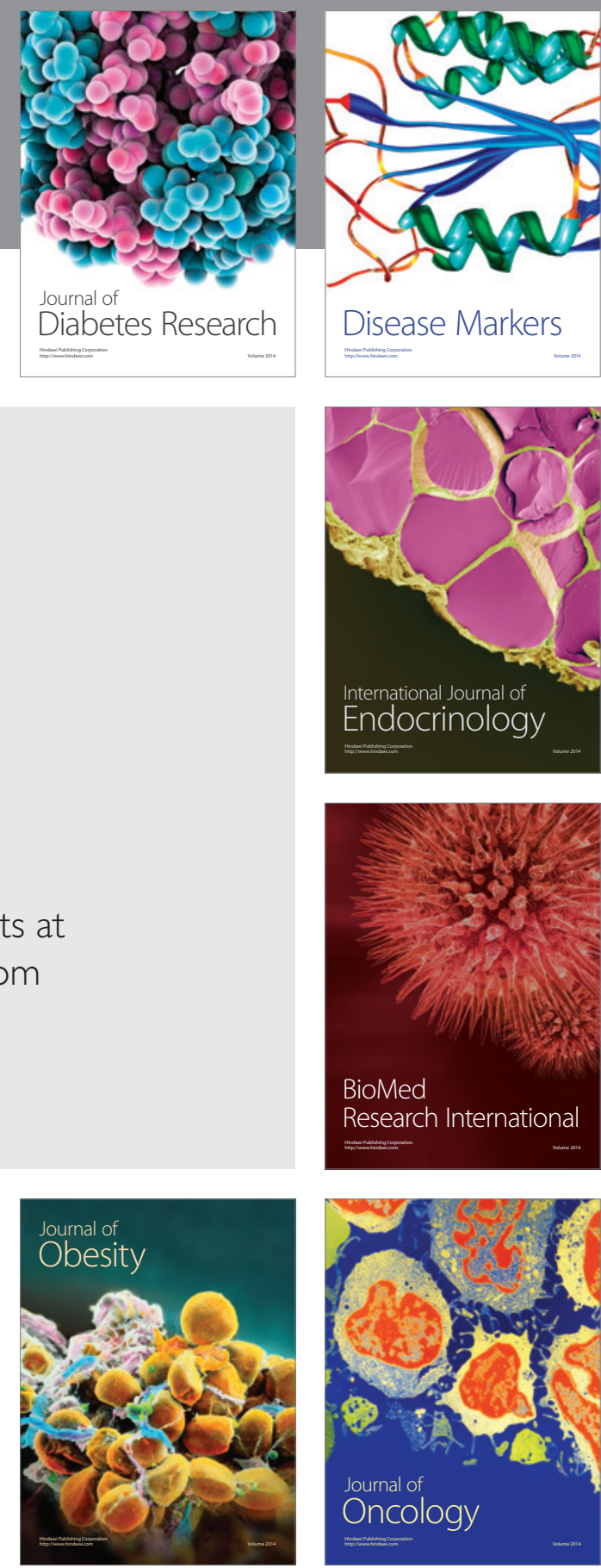

Disease Markers
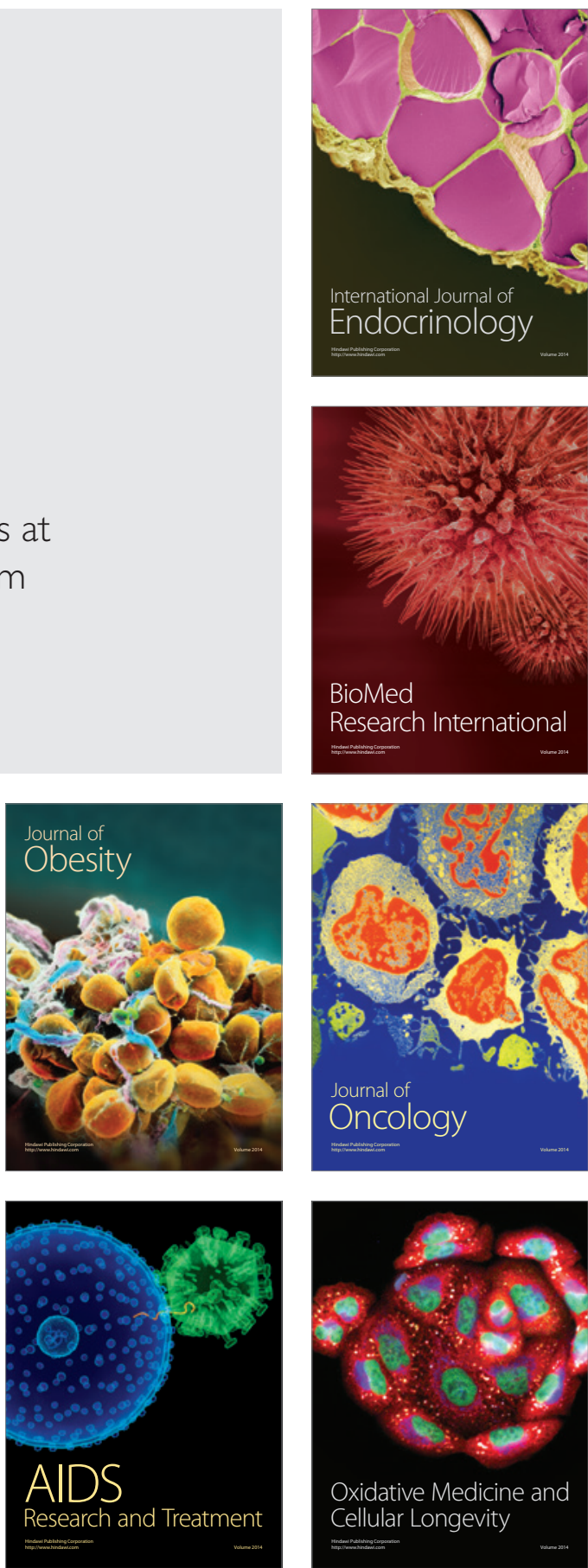